\title{
Magnetic phases from competing Hubbard and extended Coulomb interactions in twisted bilayer graphene
}

\author{
J. González $\oplus^{1}$ and T. Stauber ${ }^{2}$ \\ ${ }^{1}$ Instituto de Estructura de la Materia, CSIC, E-28006 Madrid, Spain \\ ${ }^{2}$ Materials Science Factory, Instituto de Ciencia de Materiales de Madrid, CSIC, E-28049 Madrid, Spain
}

(Received 19 April 2021; revised 18 June 2021; accepted 17 August 2021; published 7 September 2021)

\begin{abstract}
We implement a self-consistent Hartree-Fock approximation based on a microscopic model in real space, which allows us to consider the interplay between the Hubbard and the extended Coulomb interaction in twisted bilayer graphene at the magic angle. These two interactions tend to favor different symmetry-breaking patterns, therefore having complementary roles in the regimes where one or the other dominates. We show that, for sufficiently large values of the on-site Hubbard repulsion, magic angle graphene has an antiferromagnetic ground state at the charge neutrality point, while at half filling of the lowest valence band the state becomes fully spin-polarized. In general, a suitable screening of the extended Coulomb interaction is required to observe the magnetic state in either case, as otherwise the instabilities take place in the charge sector, preferentially in the form of time-reversal, chiral, or valley symmetry breaking.
\end{abstract}

DOI: 10.1103/PhysRevB.104.115110

\section{INTRODUCTION}

The discovery of superconductivity and correlated phases in twisted bilayer graphene (TBG) at the so-called magic angle [1,2] has opened a new era in the investigation of strong electron correlations in layered materials [3-33]. There is indeed evidence that those phenomena may arise as a consequence of the strong electron-electron interaction, drawing a possible connection with the unconventional behavior of the copper-oxide superconductors $[34,35]$.

Some of the most prominent effects in TBG have to do with the opening of a gap in the electronic spectrum at integer fillings of the lowest valence and conduction bands $[1,2,6]$. It has been remarkable the observation of ferromagnetism at three-quarter filling of the lowest conduction band, with the concomitant breakdown of the spin and valley symmetries of the bilayer [36]. The gap seen at the charge neutrality point (CNP) is also likely to arise from a dynamical breakdown of symmetry, by which the strong electronic interaction would destabilize the Dirac nodes in the spectrum.

There have been experimental [37-39] as well as theoretical $[31,37,40-52]$ studies showing the feasibility that the electron interactions may induce different symmetry-breaking patterns in TBG at the magic angle, although these have been mainly limited to the charge sector. Magnetic instabilities have also been analyzed [53-57] but focusing the discussion on the spin sector, perhaps due to the difficulty in dealing with a microscopic model discerning spin-dependent versus spinindependent interactions. In this regard, the relative strength of the spin-dependent Hubbard interaction (as compared to that of the extended Coulomb interaction) is the key element which governs the possibility of having a spin instability at different filling factors in TBG.

In this paper, we implement a self-consistent Hartree-Fock approximation based on a microscopic model in real space, which allows us to consider the interplay between the Hubbard and the extended Coulomb interaction in TBG. These two interactions tend to favor different symmetry-breaking patterns, therefore having complementary roles in the regimes where one or the other dominates. We show, in particular, that, for sufficiently large values of the on-site Hubbard repulsion and close to the magic angle, TBG has an antiferromagnetic ground state at the CNP, while at half filling of the lowest valence band the state becomes fully spin polarized as predicted by a general theorem on the flat-band Hubbard model [58-61]. In general, a suitable screening of the extended Coulomb interaction is required to observe the magnetic state in either case, as otherwise the instabilities take place in the charge sector, preferentially in the form of time-reversal, chiral, or valley symmetry breaking.

\section{HARTREE-FOCK APPROXIMATION}

We are going to focus our discussion on a twisted bilayer belonging to the set of commensurate superlattices with twist angle $\theta_{i}=\arccos \left[\left(3 i^{2}+3 i+0.5\right) /\left(3 i^{2}+3 i+1\right)\right][62,63]$, taking, in particular, the representative with $i=28$ (twist angle $\theta \approx 1.16^{\circ}$ ). The Hamiltonian $H$ can be written as the sum of a noninteracting piece $H_{0}$ and the term $H_{\text {int }}$ containing the Hubbard and Coulomb interactions:

$$
H=H_{0}+H_{\text {int }} .
$$

We represent $H_{0}$ in the form of a tight-binding Hamiltonian, adopting the parametrization described in the Appendix. In the moiré superlattice with electrons sitting at lattice sites $\boldsymbol{r}_{i}$ with spin $\sigma=\uparrow, \downarrow$, the matrix representation $\left(H_{0}\right)_{i \sigma, j \sigma}$ can be exactly diagonalized, leading to eigenvalues $\varepsilon_{a \sigma}^{0}$ and eigenvectors $\phi_{a \sigma}^{0}\left(\boldsymbol{r}_{i}\right)$. In the zero-frequency (static) limit, the noninteracting electron propagator $G_{0}$ is just the inverse of $H_{0}$ 
and can be written as

$$
\left(G_{0}\right)_{i \sigma, j \sigma}=-\sum_{a} \frac{1}{\varepsilon_{a \sigma}^{0}} \phi_{a \sigma}^{0}\left(\boldsymbol{r}_{i}\right) \phi_{a \sigma}^{0}\left(\boldsymbol{r}_{j}\right)^{*} .
$$

The Hartree-Fock approximation relies on the assumption that the full electron propagator $G$ can be represented in terms of a modified set of eigenvalues $\varepsilon_{a \sigma}$ and eigenvectors $\phi_{a \sigma}\left(\boldsymbol{r}_{i}\right)$, in such a way that in the static limit

$$
(G)_{i \sigma, j \sigma}=-\sum_{a} \frac{1}{\varepsilon_{a \sigma}} \phi_{a \sigma}\left(\boldsymbol{r}_{i}\right) \phi_{a \sigma}\left(\boldsymbol{r}_{j}\right)^{*}
$$

Furthermore, the relation between $G$ and $G_{0}$ is given by the electron self-energy $\Sigma$ according to the Dyson equation:

$$
G^{-1}=G_{0}^{-1}-\Sigma \text {. }
$$

In the Hartree-Fock approximation, the many-body diagrammatics implies that $\Sigma$ can be expressed in terms of the set of $\phi_{a \sigma}\left(\boldsymbol{r}_{i}\right)$. It turns out that, in the static limit,

$$
\begin{aligned}
(\Sigma)_{i \sigma, j \sigma}= & 2 \mathbb{I}_{i j} \sum_{a} \sum_{l, \sigma^{\prime}} v_{\sigma \sigma^{\prime}}\left(\boldsymbol{r}_{i}-\boldsymbol{r}_{l}\right)\left|\phi_{a \sigma^{\prime}}\left(\boldsymbol{r}_{l}\right)\right|^{2} \\
& -v_{\sigma \sigma}\left(\boldsymbol{r}_{i}-\boldsymbol{r}_{j}\right) \sum_{a}^{\prime} \phi_{a \sigma}\left(\boldsymbol{r}_{i}\right) \phi_{a \sigma}\left(\boldsymbol{r}_{j}\right)^{*},
\end{aligned}
$$

where $v_{\sigma \sigma^{\prime}}(\boldsymbol{r})$ is the interaction potential between electron densities with spin $\sigma$ and $\sigma^{\prime}$ and the prime means that the sum is to be carried over the occupied levels [64].

The problem of finding the set of $\varepsilon_{a \sigma}$ and $\phi_{a \sigma}\left(\boldsymbol{r}_{i}\right)$ amounts then to solving the self-consistent equation given by Eqs. (4) and (5). This can be achieved in practice by means of a recursive procedure, in which the self-energy is built at each step from the approximate eigenvectors obtained in the previous iteration (see the Appendix for details of the computation).

One of the advantages of applying the Hartree-Fock approximation in real space is the possibility to discern the contribution of different interactions to the potential $v_{\sigma \sigma^{\prime}}(\boldsymbol{r})$. We have, for instance, a term $H_{\mathrm{C}}$ in the interaction Hamiltonian corresponding to the extended Coulomb interaction, which we will take as suitably screened by nearby metallic gates to make contact with typical experimental setups. Thus, we can express in terms of electron creation (annihilation) operators $a_{i \sigma}^{+}\left(a_{i \sigma}\right)$,

$$
H_{\mathrm{C}}=\frac{1}{2} \sum_{i, j, \sigma, \sigma^{\prime}} a_{i \sigma}^{\dagger} a_{i \sigma} v_{\mathrm{C}}\left(\boldsymbol{r}_{i}-\boldsymbol{r}_{j}\right) a_{j \sigma^{\prime}}^{\dagger} a_{j \sigma^{\prime}},
$$

where we take the potential appropriate to the case of top and bottom metallic gates [65], each at a distance $d=\xi / 2$ from the twisted bilayer:

$$
v_{\mathrm{C}}(\boldsymbol{r})=\frac{e^{2}}{4 \pi \epsilon} \frac{2 \sqrt{2} e^{-\pi r / \xi}}{\xi \sqrt{r / \xi}} .
$$

Moreover, we also take into account the interaction coming from the on-site repulsion of electrons sitting at the same carbon atom. This contributes to $H_{\text {int }}$ with the Hubbard term

$$
H_{\mathrm{U}}=U \sum_{i} a_{i \uparrow}^{\dagger} a_{i \uparrow} a_{i \downarrow}^{\dagger} a_{i \downarrow}
$$

The term $H_{\mathrm{U}}$ can be viewed as a prescription to define the Coulomb interaction in the limit $\boldsymbol{r} \rightarrow 0$, which cannot be obtained from Eq. (7). The on-site repulsion $U$ is actually a very relevant parameter in the subsequent discussion since the Hubbard term is the spin-dependent part of the interaction. The prevalence of the magnetic phases turns out to be dictated then by the value of $U$, as we see in what follows.

\section{SYMMETRY BREAKING AT THE CHARGE NEUTRALITY POINT}

The most distinctive experimental feature observed at the CNP of TBG at the magic angle is the opening of a gap in the electronic spectrum. This can be attributed to the effect of dynamical symmetry breaking which, for sufficiently strong electron-electron interaction, destabilizes the Dirac nodes at the $K$ points of the moiré Brillouin zone. Such an effect typically proceeds through the development of a staggered density in the charge or the spin sector. The different symmetrybreaking patterns can be built from the matrix elements

$$
h_{i j}^{(\sigma)}=\sum_{a}^{\prime} \phi_{a \sigma}\left(\boldsymbol{r}_{i}\right) \phi_{a \sigma}\left(\boldsymbol{r}_{j}\right)^{*}
$$

where the prime means again that the sum is only over occupied states. In TBG, we have sublattices $A_{1}, B_{1}$ for the top carbon layer and $A_{2}, B_{2}$ for the bottom layer. Thus, we have the order parameters:

$$
C_{ \pm \sigma}=\sum_{i \in A_{1}} h_{i i}^{(\sigma)}-\sum_{i \in B_{1}} h_{i i}^{(\sigma)} \pm\left(\sum_{i \in A_{2}} h_{i i}^{(\sigma)}-\sum_{i \in B_{2}} h_{i i}^{(\sigma)}\right) .
$$

The condensation of a staggered spin density (signaling antiferromagnetic order) may be characterized by a nonvanishing value of $C_{+\uparrow}-C_{+\downarrow}$, while the prevalence of a nonzero value of $C_{+\uparrow}+C_{+\downarrow}$ is instead the signature of staggered charge order, with the consequent chiral symmetry breaking.

As already pointed out, the Hubbard term is the only source of spin-dependent interaction in our model, so the balance between the charge and the spin order is governed by the relative strength of the on-site repulsion $U$. This quantity is assumed to have a value of the order of $\sim 8 \mathrm{eV}$ in graphene, but in TBG it may be significantly reduced due to internal screening from the narrow bands. We have estimated this effect by taking into account the relevant electron-hole excitations in the random-phase approximation (RPA), finding that a realistic coupling is given in our model by $U \approx 4 \mathrm{eV}$ (see the Appendix for a detailed evaluation). We will consider this effective value in what follows, but comparing also the results with the instance of a much smaller (unrealistic) coupling $U=0.5 \mathrm{eV}$, just for the sake of discerning the role played by the Hubbard interaction to trigger the magnetic instabilities.

For the effective value $U=4 \mathrm{eV}$, we find that the development of a staggered spin density (antiferromagnetic order) prevails over chiral symmetry breaking, regardless of the strength of the Coulomb interaction. This is shown in the phase diagram of Fig. 1(a), where the $x$ axis represents the strength of the potential Eq. (7), for a setup with $\xi=10$ nm. We observe that the antiferromagnetic phase is preserved down the limit of a pure Hubbard interaction $(\epsilon \rightarrow \infty)$. 

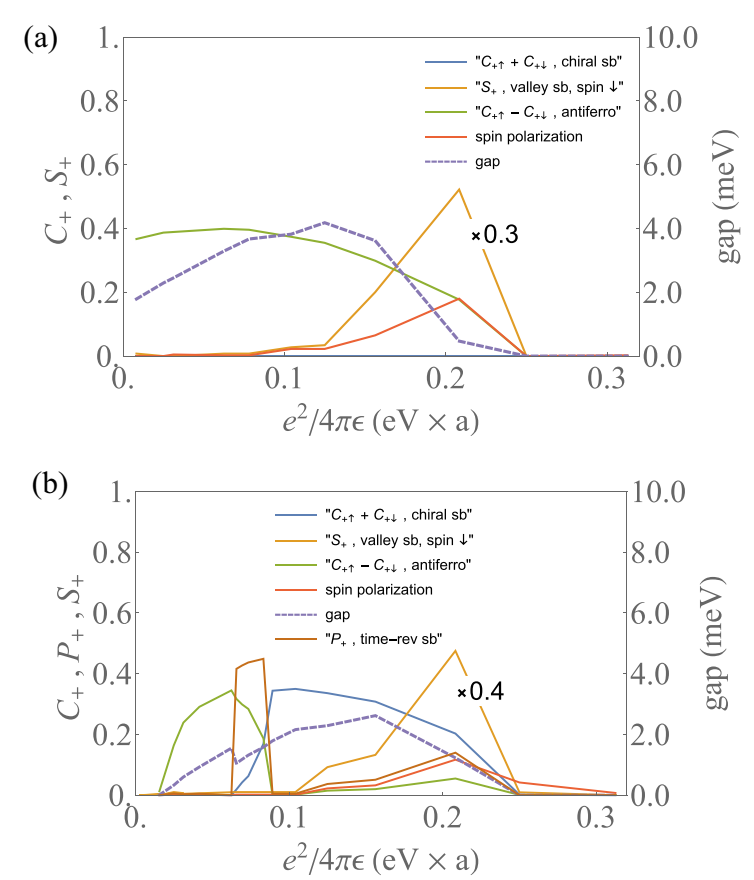

FIG. 1. Phase diagrams showing the order parameters of the dominant symmetry-breaking patterns at the charge neutrality point of twisted bilayer graphene with $i=28$ (twist angle $\theta \approx 1.16^{\circ}$ ) for two different values of the on-site Hubbard coupling $U=4.0 \mathrm{eV}$ (a) and $0.5 \mathrm{eV}$ (b). The $x$ axis corresponds to the coupling of the Coulomb potential (in units where $a$ is the $\mathrm{C}-\mathrm{C}$ distance).

When the antiferromagnetic signal decreases to the right of the diagram in Fig. 1(a), we find, however, the onset of a different order parameter corresponding to valley symmetry breaking in TBG. This may be characterized by the circulation of the matrix elements $h_{i j}$ along the loop of the three nearest-neighbors $i_{1}, i_{2}$, and $i_{3}$ of each atom $i$, with clockwise orientation. We may have a nonvanishing flux inside each loop indicating the breakdown of time-reversal invariance (TRI) but with opposite sign in the two sublattices $A$ and $B$. This condensation is measured by the order parameters:

$$
\begin{aligned}
S_{ \pm \sigma}= & \operatorname{Im}\left(\sum_{i \in A_{1}}\left(h_{i_{1} i_{2}}^{(\sigma)} h_{i_{2} i_{3}}^{(\sigma)} h_{i_{3} i_{1}}^{(\sigma)}\right)^{\frac{1}{3}}-\sum_{i \in B_{1}}\left(h_{i_{1} i_{2}}^{(\sigma)} h_{i_{2} i_{3}}^{(\sigma)} h_{i_{3} i_{1}}^{(\sigma)}\right)^{\frac{1}{3}}\right. \\
& \left. \pm \sum_{i \in A_{2}}\left(h_{i_{1} i_{2}}^{(\sigma)} h_{i_{2} i_{3}}^{(\sigma)} h_{i_{3} i_{1}}^{(\sigma)}\right)^{\frac{1}{3}} \mp \sum_{i \in B_{2}}\left(h_{i_{1} i_{2}}^{(\sigma)} h_{i_{2} i_{3}}^{(\sigma)} h_{i_{3} i_{1}}^{(\sigma)}\right)^{\frac{1}{3}}\right) .
\end{aligned}
$$

The phase realized to the right of the diagram in Fig. 1(a) corresponds to nonvanishing $S_{+\sigma}$ for the less populated spin $\sigma$. In the continuum theory of Dirac fermions, this breakdown of symmetry translates into the generation of a term proportional to the identity in pseudospin space. This does not open a gap in the Dirac cones at the $K$ point but instead leads to a different shift in the energy of the cones in the two valleys of the twisted bilayer, with the consequent valley symmetry breaking.

On the other hand, if we take an effective value of $U=$ $0.5 \mathrm{eV}$, the phase diagram shows a different competition between symmetry-breaking patterns as seen in Fig. 1(b). For that value of $U$, we observe that there is no magnetic instability in the limit of a pure Hubbard interaction $\epsilon \rightarrow \infty$.
However, there is still an antiferromagnetic phase in the weakcoupling regime of the extended Coulomb interaction, where this is presumably reinforcing the Hubbard interaction to produce the staggered spin order.

For larger extended Coulomb interaction, we find next a mixed phase where there is chiral symmetry breaking (staggered charge order) for one of the spin polarizations, while for the other polarization the state corresponds to a Chern insulator with the order parameter

$$
\begin{aligned}
P_{+\sigma}= & \operatorname{Im}\left(\sum_{i \in A_{1}}\left(h_{i_{1} i_{2}}^{(\sigma)} h_{i_{2} i_{3}}^{(\sigma)} h_{i_{3} i_{1}}^{(\sigma)}\right)^{\frac{1}{3}}+\sum_{i \in B_{1}}\left(h_{i_{1} i_{2}}^{(\sigma)} h_{i_{2} i_{3}}^{(\sigma)} h_{i_{3} i_{1}}^{(\sigma)}\right)^{\frac{1}{3}}\right. \\
& \left.+\sum_{i \in A_{2}}\left(h_{i_{1} i_{2}}^{(\sigma)} h_{i_{2} i_{3}}^{(\sigma)} h_{i_{3} i_{1}}^{(\sigma)}\right)^{\frac{1}{3}}+\sum_{i \in B_{2}}\left(h_{i_{1} i_{2}}^{(\sigma)} h_{i_{2} i_{3}}^{(\sigma)} h_{i_{3} i_{1}}^{(\sigma)}\right)^{\frac{1}{3}}\right) .
\end{aligned}
$$

For a yet stronger Coulomb interaction, we turn into a phase with chiral symmetry breaking in the two spin polarizations. This phase as well as the preceding Chern insulating phase are already present in the phase diagram obtained for spin-independent interactions in TBG [47]. We may therefore interpret that those two phases reflect the regime of prevalence of the extended Coulomb interaction. In any case, we observe that there is still a residual effect of the Hubbard interaction to the right of the phase diagram in Fig. 1(b), leading to a slight spin polarization and a concomitant phase with valley symmetry breaking.

Finally, for the smallest values of the dielectric constant $\epsilon$, there is no signal of symmetry breaking in the phase diagram of Fig. 1(b). Nevertheless, this is a consequence of the fact that, for such a strong-coupling regime, the Fermi level departs at charge neutrality from the neighborhood of the Dirac nodes at the $K$ points of the moiré Brillouin zone. This explains the apparent discrepancy with the phase diagrams in Ref. [47], where the phases have been always found by constraining the Fermi level to fall between the upper and lower Dirac cones.

\section{SYMMETRY BREAKING AT HALF FILLING OF THE LOWEST VALENCE BAND}

As the magic angle is approached and the low-energy bands flatten, the one-particle density of states becomes progressively higher in the lowest valence and conduction bands of TBG. The question of symmetry breaking then becomes relevant and, in particular, whether a spin-polarized state may arise due to interaction effects. In this regard, the key role is played by the effective value after internal screening of the Hubbard coupling $U$. As in the previous section, we are going to consider again a realistic effective value of the Hubbard coupling $U=4 \mathrm{eV}$, but comparing also with the instance of a much smaller coupling $(U=0.5 \mathrm{eV})$ to show how the Hubbard interaction leads to important changes in the phase diagram, inducing a kind of rotation between SU(2)-spin and valley symmetry breaking.

For an effective on-site repulsion $U=4 \mathrm{eV}$, the Hubbard interaction is strong enough to produce a full spin polarization in the ground state of the system. This is shown in the phase diagram of Fig. 2(a), which represents the different phases for growing strength of the extended Coulomb interaction. 

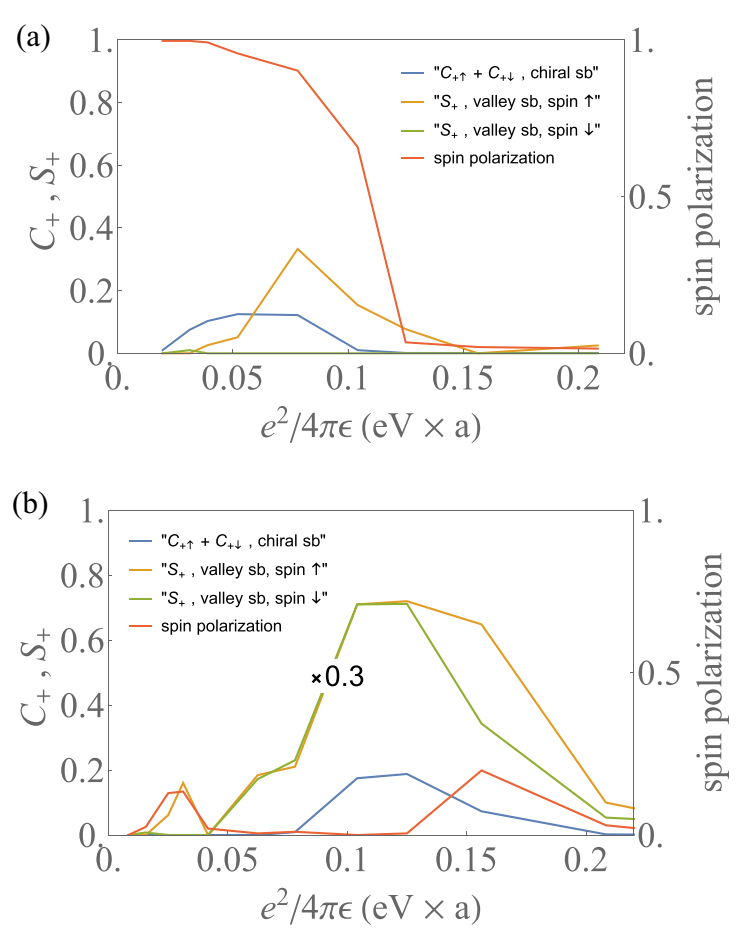

FIG. 2. Phase diagrams showing the order parameters of the dominant symmetry breaking patterns at half filling of the lowest valence band of twisted bilayer graphene with $i=28$ (twist angle $\theta \approx 1.16^{\circ}$ ) for two different values of the on-site Hubbard coupling $U=4.0 \mathrm{eV}$ (a) and $0.5 \mathrm{eV}$ (b). The $x$ axis corresponds to the coupling of the Coulomb potential (in units where $a$ is the $\mathrm{C}-\mathrm{C}$ distance).

The full spin polarization is localized around the AA-stacked region of the moiré unit cell, see Fig. 3, and takes place along with the complete splitting of the low-energy bands for spin up and spin down. This means that, at half filling, the polarized electrons populate the states up to the level of the Dirac nodes of the filled band. These are then susceptible of being destabilized by the electronic interaction, as actually happens with the development of chiral symmetry breaking seen in the phase diagram of Fig. 2(a). The diagram also shows a clear competition between the effects of the Hubbard and the extended Coulomb interaction, which leads to the suppression of the spin polarization for sufficiently small values of the dielectric constant $\epsilon$.

The same effect of full spin polarization is found for $U=$ $2 \mathrm{eV}$ in the limit $\epsilon \rightarrow \infty$, but for $U=0.5 \mathrm{eV}$ the magnetic ground state is lost, as shown in Fig. 2(b). For that value of $U$, we also observe that the main signature of symmetry breaking corresponds to the prominent growth of the order parameter $S_{+}$defined in Eq. (11). Therefore, the main physical effect is that of valley symmetry breaking, which is driven by the extended Coulomb interaction and grows large for small values of the dielectric constant.

\section{CONCLUSION}

In this paper, we have reported self-consistent HartreeFock calculations for magic angle TBG, including both on-site Hubbard and extended Coulomb interactions. We have found that the former favors an antiferromagnetic ground state at the CNP, except in cases with relatively small Hubbard coupling, where the dynamical generation of a gap would be mainly due to the extended Coulomb interaction. Let us remark that we have carried out the calculations without allowing for the breakdown of the threefold rotational symmetry of the twisted bilayer. Nevertheless, the presented conclusions are relevant because a nematic instability does not usually open a gap at the Dirac cones. This means that some of the symmetry
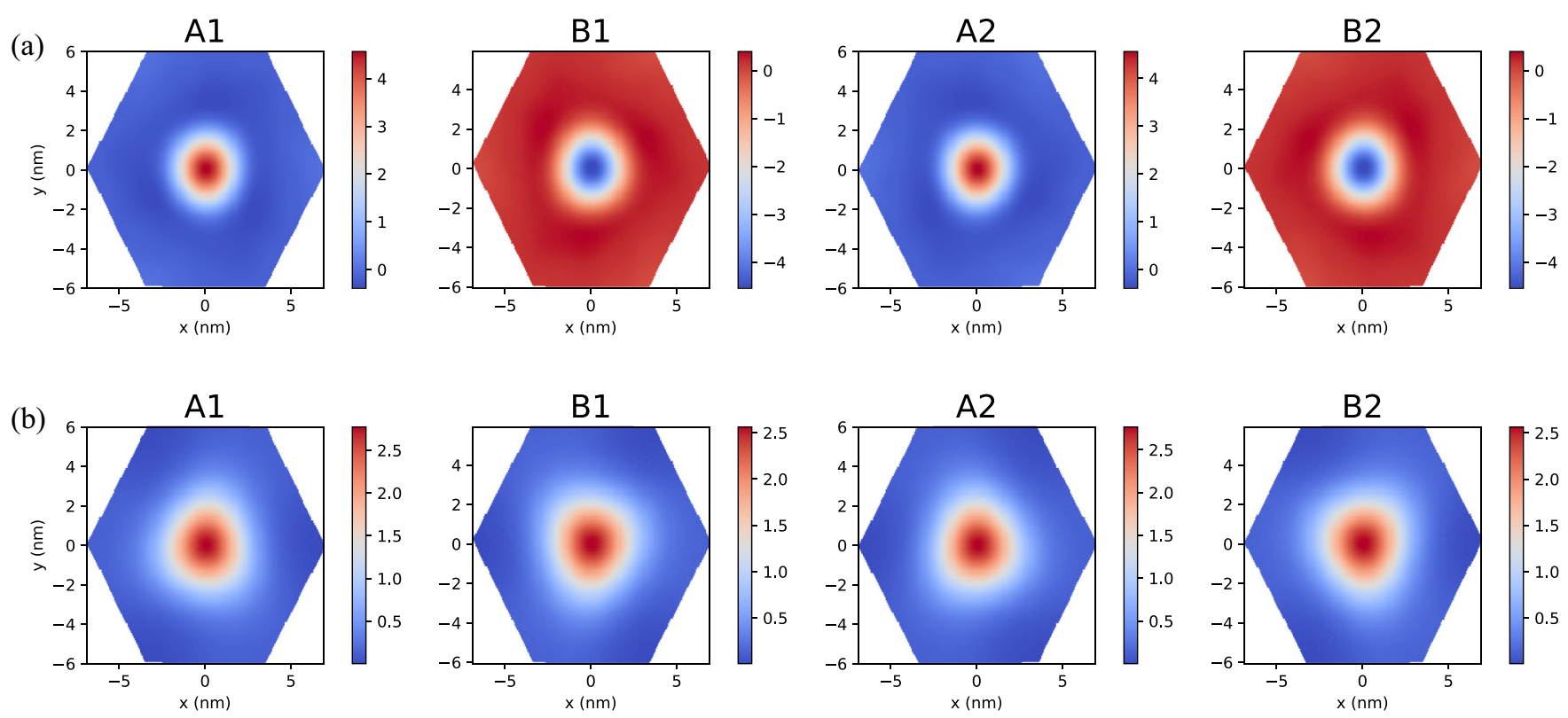

FIG. 3. Spin density at the neutrality point (a) and at half filling of the lowest valence band (b) in the hexagonal moiré unit cell of twisted bilayer graphene with $i=28$ (twist angle $\theta \approx 1.16^{\circ}$ ) for an on-site Hubbard coupling $U=4.0 \mathrm{eV}$. We show the spin densities separately for the $X$-lattice sites of layer $n(X n)$ with $X=A, B$ and $n=1,2$, in units of the density $1 / A_{i}$ of one electron inside the moiré unit cell with $A_{i}=\left(3 i^{3}+3 i+1\right) \bar{a}^{2}$ ( $\bar{a}$ being the lattice constant of graphene). 
breaking patterns discussed in the paper must be at work to produce the opening of the gap observed at the CNP.

We have been mainly interested in magnetic phases, and we have found a crossover from antiferromagnetic to ferromagnetic order when doping the system from the CNP to half filling of the first valence band for sufficiently large Hubbard interaction, i.e., for values beyond the semimetal to antiferromagnetic Mott insulator transition. The resulting spin densities are localized around the AA-stacked regions and for the half-filled valence band, they can be as large as ten times the density of one electron per moiré unit cell. We thus believe that this magnetic lattice should be detectable in future experiments, providing a way of testing the balance between Hubbard and extended Coulomb interactions in the twisted bilayer.

\section{ACKNOWLEDGMENTS}

We thank Heiner Kohler for helpful discussions. This work has been supported by MINECO (Spain) under Grant No. FIS2017-82260-P, MICINN (Spain) under Grant No. PID2020-113164GB-I00, as well as by the CSIC Research Platform on Quantum Technologies PTI-001. The access to computational resources of CESGA (Centro de Supercomputación de Galicia) is also gratefully acknowledged.

\section{APPENDIX}

\section{Geometry of twisted bilayer graphene}

In TBG, a Moiré pattern appears which exhibits the same hexagonal lattice structure as single layer graphene. Depending on the twist angle between the two layers, the length of the lattice vectors of this superstructure might be largely enhanced as compared to $a \approx 2.42 \AA$, the length of the lattice vectors $\mathbf{a}_{1,2}=a( \pm 1 / 2, \sqrt{3} / 2)$ of graphene monolayer. Strictly speaking, not all twist angles are allowed but only a commensurate set [66] $\theta_{m n}$ which map the lattice point $\left(m \mathbf{a}_{1}, n \mathbf{a}_{2}\right)$ onto $\left(n \mathbf{a}_{1}, m \mathbf{a}_{2}\right), m, n \in \mathbb{N}$. However, the set of possible angles is dense in $[0,2 \pi]$ such that that spectral properties at low energies depend continuously on the value of the angle only and not on the integers $n, m$. In the following, we focus on $m-n=1$, obtaining a discrete set of twist angles $\theta_{i}$ which are labeled by only one integer $i$ through

$$
\cos \left(\theta_{i}\right)=1-\frac{1}{2 A_{i}}, \quad A_{i}=3 i^{2}+3 i+1 .
$$

An arbitrary small twist angle can be achieved by increasing the integer $i$ keeping in mind that all intermediate angles can be approximated to arbitrary accuracy by allowing two integers. The lattice vectors of the superlattice are

$$
\mathbf{t}_{1}=i \mathbf{a}_{1}+(i+1) \mathbf{a}_{2}, \quad \mathbf{t}_{2}=-(i+1) \mathbf{a}_{1}+(2 i+1) \mathbf{a}_{2},
$$

spanning a super unit cell with an area, which is $A_{i}$ times large than the unit cell of single layer graphene. Likewise, the area of the reciprocal superlattice spanned by the vectors

$$
\begin{aligned}
& \mathbf{g}_{1}=\frac{1}{A_{i}}\left((2 i+1) \mathbf{b}_{1}+(i+1) \mathbf{b}_{2}\right), \\
& \mathbf{g}_{2}=\frac{1}{A_{i}}\left(-(i+1) \mathbf{b}_{1}+i \mathbf{b}_{2}\right),
\end{aligned}
$$

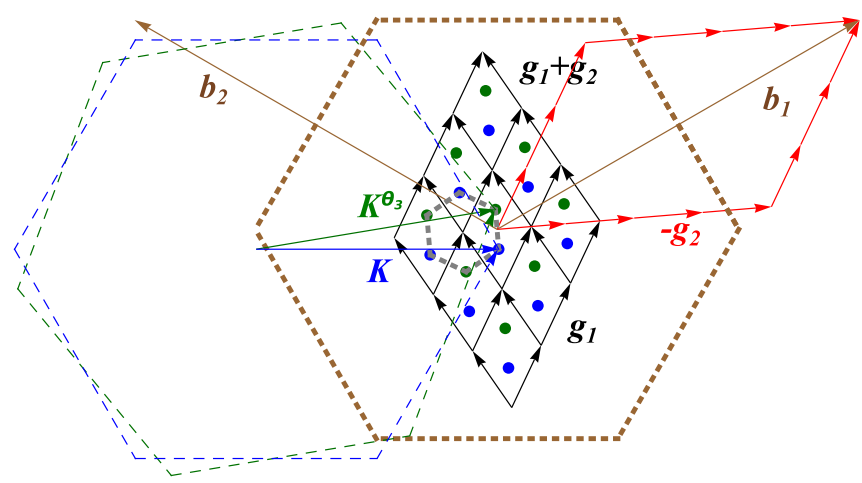

FIG. 4. Sketch of the twisted bilayer in $k$ space for a large angle $i=3$. The Brillouin zones of the two layers (dashed green and blue) are rotated by an angle $\theta_{3} \approx 9.14^{\circ}$. The Dirac points $\mathbf{K}$ and $\mathbf{K}^{\theta_{i}}$ are the two inequivalent Dirac points of the Brillouin zone of the superlattice (small dashed grey hexagon). The lattice vectors $\mathbf{b}_{1,2}$ of the reciprocal lattice of graphene monolayer (brown arrows) are related to the lattice vectors of the reciprocal superlattice (red arrows) by $\mathbf{b}_{1}=i \mathbf{g}_{1}-(i+1) \mathbf{g}_{2}$.

is $A_{i}$ times smaller than the area of the reciprocal lattice of graphene, spanned by $\mathbf{b}_{1,2}=2 \pi( \pm 1 / a, 1 /(a \sqrt{3}))$. The Brillouin zones of the two monolayers are twisted by the angle $\theta_{i}$, such that the Dirac points $\mathbf{K}^{\phi}, \phi=0, \theta_{i},\left(\mathbf{K} \equiv \mathbf{K}^{0}\right)$ at the positions $\mathbf{K}^{\phi}=(4 \pi / 3)(\cos \phi, \sin \phi)$ are connected by the vector $\Delta \mathbf{K} \equiv \mathbf{K}^{\theta_{i}}-\mathbf{K}=\left(2 \mathbf{g}_{1}+\mathbf{g}_{2}\right) / 3$, which shrinks as the twist angle $\theta_{i}$ becomes increasingly smaller $|\Delta \mathbf{K}|=|\mathbf{K}| / \sqrt{A_{i}}$. The two points $\mathbf{K}$ and $\mathbf{K}^{\theta_{i}}$ are the vertices of the Brillouin zone of the reciprocal superlattice with the $\Gamma$ point at $\mathbf{K}^{\theta_{i}}+\left(\mathbf{g}_{1}+\right.$ $\left.\mathbf{g}_{2}\right) / 3$ and one $M$ point at $\mathbf{M}=\left(\mathbf{K}+\mathbf{K}^{\theta_{i}}\right) / 2$. The geometry in momentum space is summarized in Fig. 4.

\section{Tight-binding model for twisted bilayer graphene}

Modeling TBG in a tight-binding approach, we take as the starting point the noninteracting Hamiltonian:

$$
\begin{aligned}
H_{0}= & -\sum_{\langle i, j\rangle} t_{\|}\left(\boldsymbol{r}_{i}-\boldsymbol{r}_{j}\right)\left(a_{i \sigma}^{\dagger} a_{j \sigma}+\text { H.c. }\right) \\
& -\sum_{(i, j)} t_{\perp}\left(\boldsymbol{r}_{i}-\boldsymbol{r}_{j}\right)\left(a_{i \sigma}^{\dagger} a_{j \sigma}+\text { H.c. }\right) .
\end{aligned}
$$

The sum over the brackets $\langle\ldots\rangle$ runs over pairs of atoms in the same layer (1 or 2), whereas the sum over the curved brackets (...) runs over pairs with atoms belonging to different layers. $t_{\|}(\boldsymbol{r})$ and $t_{\perp}(\boldsymbol{r})$ are hopping matrix elements which have an exponential decay with the distance $|\boldsymbol{r}|$ between carbon atoms. A common parametrization is based on the Slater-Koster formula for the transfer integral [67],

$$
-t(\mathbf{d})=V_{p p \pi}(d)\left[1-\left(\frac{\mathbf{d} \cdot \boldsymbol{e}_{z}}{d}\right)^{2}\right]+V_{p p \sigma}(d)\left(\frac{\mathbf{d} \cdot \boldsymbol{e}_{z}}{d}\right)^{2}
$$

with

$$
\begin{aligned}
& V_{p p \pi}(d)=V_{p p \pi}^{0} \exp \left(-\frac{d-a_{0}}{r_{0}}\right), \\
& V_{p p \sigma}(d)=V_{p p \sigma}^{0} \exp \left(-\frac{d-d_{0}}{r_{0}}\right),
\end{aligned}
$$




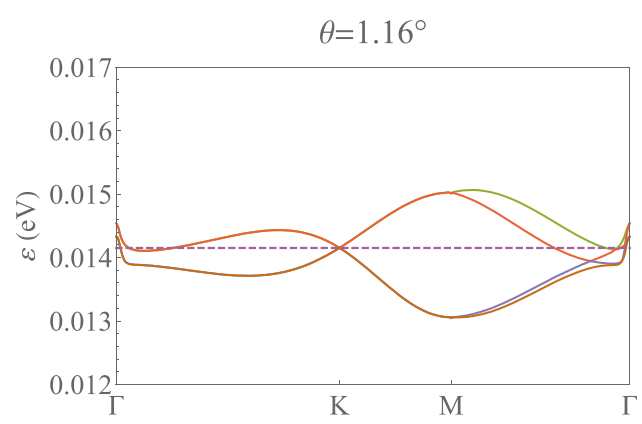

(a)

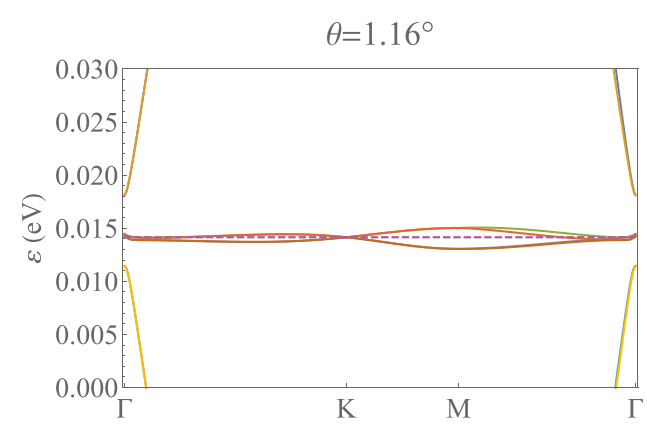

(b)

FIG. 5. Dispersion of the first valence and conduction bands [zoomed out in (b)] about the charge neutrality point of a twisted graphene bilayer with twist angle $\theta \approx 1.16^{\circ}$, computed in a tight-binding approach with parameters given in the text.

where $\mathbf{d}$ is the vector connecting the two sites, $\boldsymbol{e}_{z}$ is the unit vector in the $z$-direction, $a_{0}$ is the $\mathrm{C}$-C distance and $d_{0}$ is the distance between layers. A typical choice of parameters is given by $V_{p p \pi}^{0}=-2.7 \mathrm{eV}, V_{p p \sigma}^{0}=0.48 \mathrm{eV}$, and $r_{0}=0.319 a_{0}$ [67]. In practice, we have taken these values to carry out the analysis reported in the main text, but restricting within each layer the hopping to nearest-neighbor atoms, as a way to get closer to the magic angle condition. This leads to sensible results for the commensurate lattice studied in the main text with twist angle $\theta \approx 1.16^{\circ}$, whose first valence and conduction bands are shown in Fig. 5. The reduced bandwidth allows us to address the problem of symmetry breaking covering the regimes of both strong and weak electron correlation, as done in the paper by varying the screening of the Coulomb interaction.

Regarding the Hartree-Fock approximation, the construction of the self-energy in Eq. (5) of the main text demands the knowledge of the eigenvectors of the Hamiltonian. That selfenergy implies a sum over the occupied states of the electronic bands, but in practice one has to impose some kind of truncation when carrying out the calculation. In this respect, we have retained the first 64 valence bands below those hosting the Dirac nodes in the self-consistent resolution for the two values of $U$ taken at the CNP, as well as for $U=0.5 \mathrm{eV}$ at half filling of the first two valence bands. When computing in this latter instance with $U=4 \mathrm{eV}$, we have extended the number of valence bands retained up to 128 . In all cases, we have computed the self-energy taking a grid with 300 momenta (plus the gamma point) covering the Brillouin zone. We have checked that such a content of filled states is safe to capture the different symmetry-breaking patterns in the phase diagrams, for the interaction strength considered in the paper. We stress that the choice of a sufficiently large Fermi sea of filled states is particularly crucial to unveil the antiferromagnetic order at the CNP, which does not appear when computing with a small number of valence bands but certainly becomes the dominant pattern in any sensible calculation at large $U$, as shown in the main text.

\section{Continuum model for twisted bilayer graphene}

For the continuum model, we follow Refs. [62,63] and take into account interlayer hopping only between the two sites in each layer which are closest to each other. The hopping amplitude $t(\mathbf{R}, \boldsymbol{\delta})$ between a lattice site $\mathbf{R}$ on the first layer and site $\mathbf{R}+\boldsymbol{\delta}+d \mathbf{e}_{3}$ of the second one is much smaller than the intralayer hopping amplitude $t_{0} \approx 2.78 \mathrm{eV}$. In principle, $t(\mathbf{R}, \boldsymbol{\delta})$ has a rather complicated space dependence but its periodicity with respect to the superlattice allows an expansion in Fourier components $\tilde{t}_{\sigma \sigma^{\prime}}(\mathbf{g})$. These depend on a reciprocal vector of the superlattice $\mathbf{g}$ and on whether the hopping takes place between a $\sigma=A, B$ sublattice of the first layer and a $\sigma^{\prime}=A^{\prime}, B^{\prime}$ sublattice on the second layer. Using the Dirac cone approximation for single layer graphene, the tightbinding Hamiltonian for twisted graphene reads close to the Dirac point $\mathbf{K}[68,69] \hat{H}=\sum_{\mathbf{k}} \hat{H}(\mathbf{k})$ and

$$
\begin{aligned}
\hat{H}(\mathbf{k})= & \hbar v_{\mathrm{F}} \sum_{\phi=0, \theta_{i}} \hat{a}_{\phi A \mathbf{k}}^{\dagger} \hat{\boldsymbol{\sigma}}_{\mathrm{AB}}^{\phi} \cdot\left(\mathbf{k}+\mathbf{M}-\mathbf{K}^{\phi}\right) \hat{a}_{\phi B \mathbf{k}} \\
& +\sum_{\sigma, \sigma^{\prime}, \mathbf{g}} \hat{a}_{0 \sigma \mathbf{k}+\mathbf{g}}^{\dagger} \tilde{t}_{\sigma \sigma^{\prime}}(\mathbf{g}) \hat{a}_{\theta_{i} \sigma^{\prime} \mathbf{k}}+\text { H.c. }
\end{aligned}
$$

Here $\hat{a}_{\phi \sigma \mathbf{k}}^{\dagger}$ creates an electron on a single layer with twist angle $\phi$ on the sublattice $\sigma$ with lattice momentum $\mathbf{k}+\mathbf{M}-\mathbf{K}^{\phi}$. Moreover, in Eq. (A7) the 2D-spin vector operator $\hat{\boldsymbol{\sigma}}^{\phi}=$ $e^{i \phi \hat{\sigma}_{z} / 2} \hat{\boldsymbol{\sigma}} e^{-i \phi \hat{\sigma}_{z} / 2}, \hat{\boldsymbol{\sigma}}=\left(\hat{\sigma}_{x}, \hat{\sigma}_{y}\right)$ was defined, with Pauli matrices $\sigma_{x}, \sigma_{y}$, and $\sigma_{z}$. The Fermi velocity of a graphene monolayer is $v_{\mathrm{F}}=\sqrt{3} a t_{0} /(2 \hbar) \approx 9 \times 10^{5} \mathrm{~ms}^{-1}$. The Dirac cone approximation is expected to be a good one for values of $\mathbf{k}$ much smaller than $|\mathbf{K}|=4 \pi /(3 a)$, respectively for energies much smaller than $\hbar v_{\mathrm{F}}|\mathbf{K}| \approx 10.1 \mathrm{eV}$. In practice, we will consider frequencies in a range $\omega<\Lambda$, where $\Lambda \sim 0.8 t_{0} / \hbar$ corresponding to a cutoff wave vector $k_{\Lambda} \approx a^{-1}$.

The interlayer hopping was investigated in detail in Refs. $[62,68]$ and with a different but equivalent approach in Ref. [69]. In Ref. [62], it was pointed out that the modulus of the interlayer hopping matrix element is independent of the sublattice index $t_{\perp}(\mathbf{g})=\left|\tilde{\sigma}_{\sigma \sigma^{\prime}}(\mathbf{g})\right|$. Moreover, $t_{\perp}(\mathbf{g})$ decreases algebraically with $a A_{i}|\Delta \mathbf{K}+\mathbf{g}|$. Thus, it is well justified to consider only these values for $n, m \in \mathbb{Z}, \mathbf{g}=n \mathbf{g}_{1}+m \mathbf{g}_{2}$ for which this quantity is minimal and neglect all others. There exist three pairs of integers $(n, m)=(0,0),(-1,0),(-1,-1)$ yielding the same minimal value $t_{\perp}=t_{\perp}(0)$. Note the asymmetry in these pairs of integers, which breaks translation invariance in $\mathbf{k}$-space. The phases of $\tilde{t}_{\sigma \sigma^{\prime}}(\mathbf{g})$ were worked out in Refs. [62,69] utilizing geometric arguments $(\xi=2 \pi / 3)$,

$$
\tilde{t}(0)=t_{\perp}\left(\begin{array}{ll}
1 & 1 \\
1 & 1
\end{array}\right), \quad \tilde{t}\left(-\mathbf{g}_{1}\right)=t_{\perp}\left(\begin{array}{cc}
e^{i \xi} & 1 \\
e^{-i \xi} & e^{i \xi}
\end{array}\right),
$$


and $\tilde{t}\left(-\mathbf{g}_{1}-\mathbf{g}_{2}\right)=\tilde{t}^{*}\left(-\mathbf{g}_{1}\right)$. We choose for the interlayer hopping strength the value $t_{\perp} \approx 110 \mathrm{meV}$ in agreement with Refs. $[69,70]$. Note that the Hamiltonian Eq. (A7) is only a valid approximation close to the Dirac point $\mathbf{K}$ and breaks TRI. In a full calculation, one has to take into account both inequivalent Dirac points. Let us call $\hat{H}^{\prime}(\mathbf{k})$ the Hamiltonian close to the second Dirac point $\mathbf{K}^{\prime}=-\mathbf{K}$. Applying the same approximations to $\hat{H}^{\prime}(\mathbf{k})$ as to $\hat{H}(\mathbf{k})$, we find $\hat{H}(\mathbf{k})=$ $\left(\hat{H}^{\prime}(-\mathbf{k})\right)^{T}$ and therefore TRI is conserved in the full Hamiltonian. Thus, it suffices to focus on a single Dirac point. Note that the Hamiltonian Eq. (A7) breaks particle-hole symmetry in contrast to the one employed by Moon et al. [67]. Let us finally note that the magic angle corresponds to $i=31$.

\section{Dynamical response function}

The dynamical density-density response function is defined as

$$
\chi\left(\mathbf{r}, \mathbf{r}^{\prime}, t\right)=\frac{1}{i \hbar} \Theta(t)\left\langle\left[\hat{n}(\mathbf{r}, t), \hat{n}\left(\mathbf{r}^{\prime}\right)\right]\right\rangle .
$$

We denote $\chi(\mathbf{q}, \omega)$ its Fourier transform in space and time. Denote $E_{m}(\mathbf{k})$ and $|m(\mathbf{k})\rangle$ eigenvalues and eigenfunctions of the Hamiltonian Eq. (A7). Then $\chi(\mathbf{q}, \omega)$ can be expressed in the long wavelength limit as [70,71]

$$
\begin{aligned}
\chi(\mathbf{q}, \omega)= & \frac{g_{\mathrm{S}}}{V} \sum_{\mathbf{k}} \sum_{\sigma= \pm} \sum_{m, n}|\langle n(\mathbf{k}+\mathbf{q}) \mid m(\mathbf{k})\rangle|^{2} \\
& \times \frac{n_{\beta}\left(E_{m}(\sigma \mathbf{k})\right)-n_{\beta}\left(E_{n}(\sigma(\mathbf{k}+\mathbf{q}))\right)}{\hbar \omega-E_{n}(\sigma(\mathbf{k}+\mathbf{q}))+E_{m}(\sigma \mathbf{k})+i \eta} .
\end{aligned}
$$

Here $n_{\beta}(x)=\left(e^{\beta(x-\mu)}+1\right)^{-1}$ is the Fermi function, $\beta=$ $\left(k_{\mathrm{B}} T\right)^{-1}$. Moreover $g_{\mathrm{S}}=2$ is the spin degeneracy. Note that the expression Eq. (A10) comprises eigenvalues and eigenstates at both inequivalent Dirac valleys, such that $\chi(\mathbf{q}, \omega)$ manifestly fulfills the usual symmetries of a response function $\operatorname{Im} \chi(\mathbf{q}, \omega)=-\operatorname{Im} \chi(\mathbf{q},-\omega), \operatorname{Re} \chi(\mathbf{q}, \omega)=\operatorname{Re} \chi(\mathbf{q},-\omega)$ and $\chi(\mathbf{q}, \omega)=\chi(-\mathbf{q},-\omega)$ due to time reversal invariance.

For frequencies $\omega \gg t_{\perp} / \hbar$, the effects of the interlayer coupling become negligible and the result for two decoupled graphene monolayer at zero temperature and zero chemical potential [72]

$$
\chi_{0}(\mathbf{q}, \omega)=\frac{-i g_{\mathrm{L}} g_{\mathrm{V}} g_{\mathrm{S}}}{16 \hbar} \frac{q^{2}}{\sqrt{\omega^{2}-v_{\mathrm{F}}^{2} q^{2}}}
$$

must be recovered $\left(g_{\mathrm{L}}=2\right.$ and $g_{\mathrm{V}}=2$ are the layer and the valley degeneracies). This result holds for vanishing coupling strength $t_{\perp}$. On the other hand, in the limit $\theta_{i} \rightarrow 0$, the response function of $A A$-stacked graphene double layer is recovered [73].

\section{Static susceptibility and permittivity at charge neutrality}

Since $\lim _{\omega \rightarrow \infty} \chi(\mathbf{q}, \omega)=\chi_{0}(\mathbf{q}, \omega)$, for the first moment of the frequency $m^{(1)}=\int_{0}^{\infty} d \omega \operatorname{Im} \chi(\mathbf{q}, \omega) \omega$ the rather general $f$-sum rule [74] holds true $m^{(1)}=\Lambda_{E} q^{2}+O\left(\Lambda_{E}^{2}\right)$. However, the static susceptibility $\chi(\mathbf{q}, 0)=m^{(-1)} / \pi$ is independent of the cutoff frequency and the complicated low-frequency spectrum of twisted graphene bilayers causes significant deviations to the result of two decoupled bilayers $\chi_{0}(\mathbf{q}, 0)=$
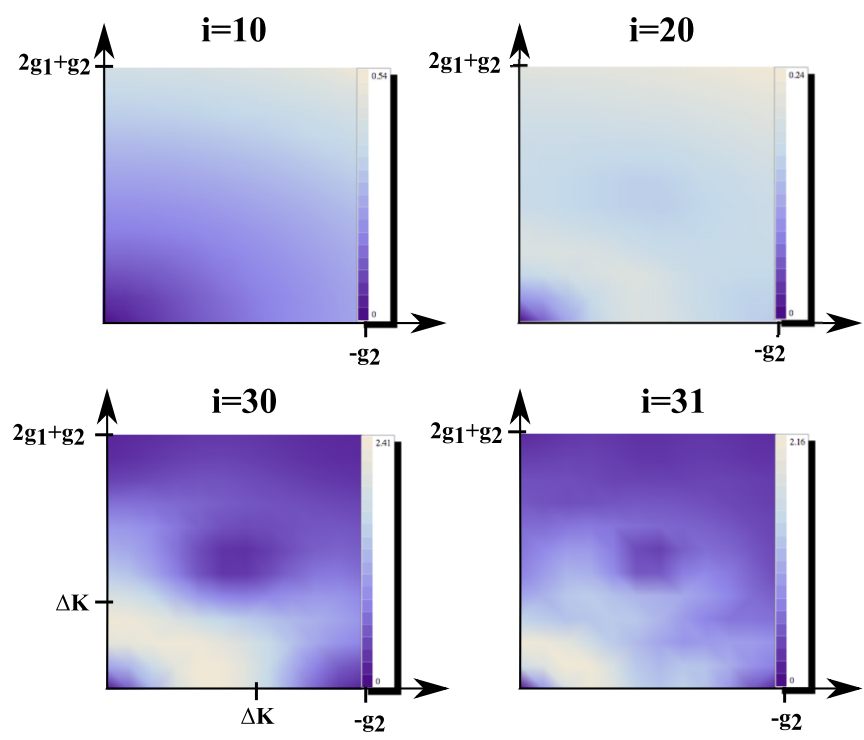

FIG. 6. Static susceptibility $\chi(\mathbf{q}, 0)$ for different twist angles as a function of the momentum transfer $\mathbf{q}$.

$q /\left(2 \hbar v_{\mathrm{F}}\right)$. This is seen in Fig. 6 , where $\chi(\mathbf{q}, \mathbf{0})$ is plotted in a density plot as a function of momentum transfer q. The domain of $\mathbf{q}$ covers the whole unit cell of the reciprocal superlattice. For large twist angles $\theta_{10}$, the static susceptibility is isotropic and monotonously increasing with $q$. However, for twist angles close to the first magic angle this behavior changes dramatically. For $\theta_{30}$, a marked maximum is visible for $\mathbf{q}$ vectors with length $q \lesssim|\Delta \mathbf{K}|$. This maximum is isotropic and at the magic angle related to the size of the moire cell. Right at the magic angle, the maximum is at a $\mathbf{q}$ vector with modulus $q \ll|\Delta \mathbf{K}|$. For $\mathbf{q}$ vectors $q>|\Delta \mathbf{K}|$, the static susceptibility becomes significantly anisotropic with a marked minimum at $\mathbf{q}=\mathbf{g}_{1}$. In Fig. 7, the static susceptibility is plotted as a function of the modulus $q$ along the direction $\Delta \mathbf{K}$ of the connection of the two Dirac points (i.e., along the $y$ axis in Fig. 6).

The maxima are most pronounced for angles close to the magic angle. The position of the maximum depends only weakly on the twist angle. The maximum of $\chi(\mathbf{q}, 0)$ at finite momentum is also manifested in the permittivity $\epsilon(\mathbf{q}, 0)$ of the material. In the RPA, the dielectric function is given by

$$
\epsilon(\mathbf{q}, \omega)=1-2 \pi \alpha_{g} \frac{\hbar v_{\mathrm{F}}}{\epsilon_{\mathrm{M} q}} \chi(\mathbf{q}, \omega),
$$

where $\alpha_{g}=e^{2} / 4 \pi \epsilon_{0} \hbar v_{\mathrm{F}} \approx 2.2$ is the fine structure constant of single layer graphene. $\epsilon_{\mathrm{M}}$ is the dielectric constant and depends on the substrate, i.e., we have $\epsilon_{\mathrm{M}} \approx 1.0$ for suspended graphene and $\epsilon_{\mathrm{M}} \approx 5.0$ for a $\mathrm{BN}$ substrate.

\section{Estimates for the internally screened Hubbard interaction}

We can estimate the value of the Hubbard interaction at charge neutrality in the following way. For an uncoupled bilayer, the dielectric function is a constant with $\epsilon_{\mathrm{BL}}=1+$ $\pi \alpha_{g} / \epsilon_{M}$. We further choose an upper band-cutoff as $\Lambda=$ $2 \pi / 1.75 \AA$ as obtained for single layer graphene [75]. With $v_{q}=\frac{e^{2}}{2 \epsilon_{0} \epsilon_{M} q}$, we have for the Hubbard parameter the following 
(a)

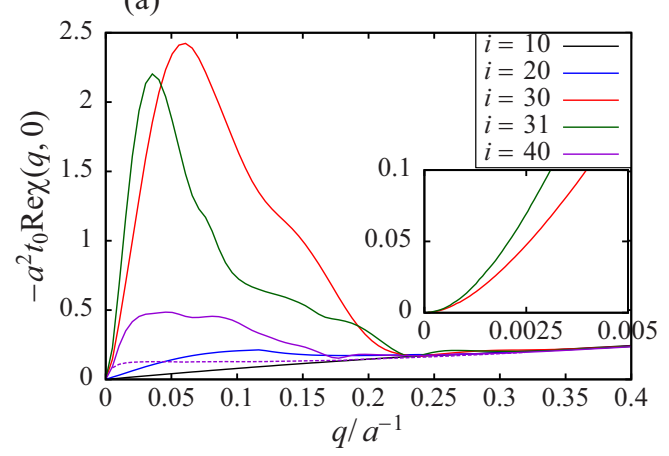

(b)

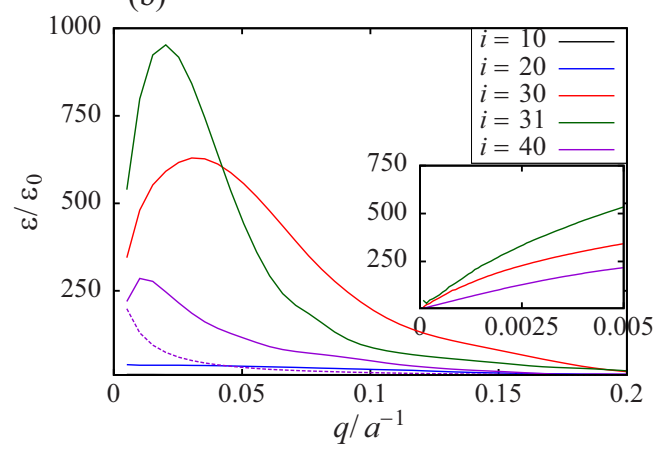

FIG. 7. Static susceptibility (a) and permittivity (b) for different twist angles $\theta_{10} \approx 3.14^{\circ}$ (black), $\theta_{20} \approx 1.61^{\circ}$ (blue), $\theta_{30} \approx 1.08^{\circ}$ (red), $\theta_{31} \approx 1.05^{\circ}$ (green), $\theta_{40} \approx 0.81^{\circ}$ (purple) as a function of $q$.

expression:

$$
U=\frac{1}{2 \pi} \int_{0}^{\Lambda} d q q \frac{v_{q}}{\epsilon(\mathbf{q}, 0)}=\frac{\alpha_{g}}{\epsilon_{M}+\pi \alpha_{g}} \hbar v_{F} \Lambda .
$$

For freestanding bilayers, taking $\hbar v_{F}=5.85 \mathrm{eV} \AA$, we then have $U=5.5 \mathrm{eV}$. Notice that this is approximately half the value of single-layer graphene, because internal screening in single layer graphene is given by $\epsilon_{\mathrm{SL}}=1+\pi \alpha_{g} / 2 / \epsilon_{M}$.

The value for $U$ is now reduced by the internal screening of TBG due to the extreme maxima of $\epsilon(\mathbf{q}, 0)$ up to $\epsilon_{m} \sim 1000$. However, the short-ranged potential is composed of larger wave numbers and the reduction can be neglected. Including the dielectric constant of $\mathrm{BN}$ with $\epsilon_{M} \sim 5$ will further reduce the nominal value and we obtain $U \sim 3.9 \mathrm{eV}$, which leads to the value used in the main text. We expect that even for finite doping close to a van Hove singularity, this value will not significantly change.

Let us finally comment on the screening of the long-ranged interaction. At the magic angle, the maximal screening is at wave number $q a \sim 0.02$ which corresponds to an intrinsic screening length of $\xi_{i} \sim 12.5 \mathrm{~nm}$ and thus approximately to the moiré lattice length. Since we assume a top and bottom gate with screening length $\xi_{g} \sim 10 \mathrm{~nm}$, we did not include this effect in our calculations. However, we predict an upper limit of an intrinsic screening length for certain gate distances.
[1] Y. Cao, V. Fatemi, S. Fang, K. Watanabe, T. Taniguchi, E. Kaxiras, and P. Jarillo-Herrero, Nature 556, 43 (2018).

[2] Y. Cao, V. Fatemi, A. Demir, S. Fang, S. L. Tomarken, J. Y. Luo, J. D. Sanchez-Yamagishi, K. Watanabe, T. Taniguchi, E. Kaxiras, R. C. Ashoori, and P. Jarillo-Herrero, Nature 556, 80 (2018).

[3] M. Yankowitz, S. Chen, H. Polshyn, Y. Zhang, K. Watanabe, T. Taniguchi, D. Graf, A. F. Young, and C. R. Dean, Science 363, 1059 (2019).

[4] E. Codecido, Q. Wang, R. Koester, S. Che, H. Tian, R. Lv, S. Tran, K. Watanabe, T. Taniguchi, F. Zhang, M. Bockrath, and C. N. Lau, Sci. Adv. 5, eaaw9770 (2019).

[5] C. Shen, Y. Chu, Q. Wu, N. Li, S. Wang, Y. Zhao, J. Tang, J. Liu, J. Tian, K. Watanabe, T. Taniguchi, R. Yang, Z. Y. Meng, D. Shi, O. V. Yazyev, and G. Zhang, Nat. Phys. 16, 520 (2020).

[6] X. Lu, P. Stepanov, W. Yang, M. Xie, M. A. Aamir, I. Das, C. Urgell, K. Watanabe, T. Taniguchi, G. Zhang, A. Bachtold, A. H. MacDonald, and D. K. Efetov, Nature 574, 653 (2019).

[7] G. Chen, A. L. Sharpe, P. Gallagher, I. T. Rosen, E. J. Fox, L. Jiang, B. Lyu, H. Li, K. Watanabe, T. Taniguchi, J. Jung, Z. Shi, D. Goldhaber-Gordon, Y. Zhang, and F. Wang, Nature 572, 215 (2019).

[8] C. Xu and L. Balents, Phys. Rev. Lett. 121, 087001 (2018).

[9] G. E. Volovik, JETP Lett. 107, 516 (2018).

[10] N. F. Q. Yuan and L. Fu, Phys. Rev. B 98, 045103 (2018).

[11] H. C. Po, L. Zou, T. Senthil, and A. Vishwanath, Phys. Rev. B 99, 195455 (2019).
[12] B. Roy and V. Juričić, Phys. Rev. B 99, 121407 (2019).

[13] H. Guo, X. Zhu, S. Feng, and R. T. Scalettar, Phys. Rev. B 97, 235453 (2018).

[14] J. F. Dodaro, S. A. Kivelson, Y. Schattner, X. Q. Sun, and C. Wang, Phys. Rev. B 98, 075154 (2018).

[15] G. Baskaran, arXiv:1804.00627.

[16] C.-C. Liu, L.-D. Zhang, W.-Q. Chen, and F. Yang, Phys. Rev. Lett. 121, 217001 (2018).

[17] K. Slagle and Y. B. Kim, SciPost Phys. 6, 16 (2019).

[18] T. J. Peltonen, R. Ojajärvi, and T. T. Heikkilä, Phys. Rev. B 98, 220504(R) (2018).

[19] D. M. Kennes, J. Lischner, and C. Karrasch, Phys. Rev. B 98, 241407(R) (2018).

[20] M. Koshino, N. F. Q. Yuan, T. Koretsune, M. Ochi, K. Kuroki, and L. Fu, Phys. Rev. X 8, 031087 (2018).

[21] J. Kang and O. Vafek, Phys. Rev. X 8, 031088 (2018).

[22] H. Isobe, N. F. Q. Yuan, and L. Fu, Phys. Rev. X 8, 041041 (2018).

[23] F. Wu, A. H. MacDonald, and I. Martin, Phys. Rev. Lett. 121, 257001 (2018).

[24] M. Ochi, M. Koshino, and K. Kuroki, Phys. Rev. B 98, 081102(R) (2018).

[25] A. Thomson, S. Chatterjee, S. Sachdev, and M. S. Scheurer, Phys. Rev. B 98, 075109 (2018).

[26] S. Carr, S. Fang, P. Jarillo-Herrero, and E. Kaxiras, Phys. Rev. B 98, 085144 (2018). 
[27] F. Guinea and N. R. Walet, Proc. Natl. Acad. Sci. USA 115, 13174 (2018).

[28] L. Zou, H. C. Po, A. Vishwanath, and T. Senthil, Phys. Rev. B 98, 085435 (2018).

[29] Y.-H. Zhang, D. Mao, Y. Cao, P. Jarillo-Herrero, and T. Senthil, Phys. Rev. B 99, 075127 (2019).

[30] J. González and T. Stauber, Phys. Rev. Lett. 122, 026801 (2019).

[31] J. Kang and O. Vafek, Phys. Rev. Lett. 122, 246401 (2019).

[32] J. M. Pizarro, M. Rösner, R. Thomale, R. Valentí, and T. O. Wehling, Phys. Rev. B 100, 161102(R) (2019).

[33] J. González and T. Stauber, Phys. Rev. Lett. 124, 186801 (2020).

[34] J. M. Park, Y. Cao, K. Watanabe, T. Taniguchi, and P. JarilloHerrero, Nature 590, 249 (2021).

[35] Z. Hao, A. M. Zimmerman, P. Ledwith, E. Khalaf, D. H. Najafabadi, K. Watanabe, T. Taniguchi, A. Vishwanath, and P. Kim, Science 371, 1133 (2021).

[36] A. L. Sharpe, E. J. Fox, A. W. Barnard, J. Finney, K. Watanabe, T. Taniguchi, M. A. Kastner, and D. GoldhaberGordon, Science 365, 605 (2019).

[37] Y. Choi, J. Kemmer, Y. Peng, A. Thomson, H. Arora, R. Polski, Y. Zhang, H. Ren, J. Alicea, G. Refael, F. von Oppen, K. Watanabe, T. Taniguchi, and S. Nadj-Perge, Nat. Phys. 15, 1174 (2019).

[38] P. Stepanov, I. Das, X. Lu, A. Fahimniya, K. Watanabe, T. Taniguchi, F. H. L. Koppens, J. Lischner, L. Levitov, and D. K. Efetov, Nature 583, 375 (2020).

[39] X. Liu, Z. Wang, K. Watanabe, T. Taniguchi, O. Vafek, and J. Li, Science 371, 1261 (2021).

[40] Y. Xie, B. Lian, B. Jäck, X. Liu, C.-L. Chiu, K. Watanabe, T. Taniguchi, B. A. Bernevig, and A. Yazdani, Nature 572, 101 (2019).

[41] T. Cea, N. R. Walet, and F. Guinea, Phys. Rev. B 100, 205113 (2019).

[42] L. Rademaker, D. A. Abanin, and P. Mellado, Phys. Rev. B 100, 205114 (2019).

[43] M. Xie and A. H. MacDonald, Phys. Rev. Lett. 124, 097601 (2020).

[44] S. Liu, E. Khalaf, J. Y. Lee, and A. Vishwanath, Phys. Rev. Res. 3, 013033 (2021).

[45] J. Liu and X. Dai, Phys. Rev. B 103, 035427 (2021).

[46] Y. Zhang, K. Jiang, Z. Wang, and F. Zhang, Phys. Rev. B 102, 035136 (2020).

[47] J. González and T. Stauber, Phys. Rev. B 102, 081118(R) (2020).
[48] J. Kang and O. Vafek, Phys. Rev. B 102, 035161 (2020).

[49] T. Cea and F. Guinea, Phys. Rev. B 102, 045107 (2020).

[50] X. Lin and J. Ni, Phys. Rev. B 102, 035441 (2020).

[51] T. Soejima, D. E. Parker, N. Bultinck, J. Hauschild, and M. P. Zaletel, Phys. Rev. B 102, 205111 (2020).

[52] O. Vafek and J. Kang, Phys. Rev. Lett. 125, 257602 (2020).

[53] L. A. Gonzalez-Arraga, J. L. Lado, F. Guinea, and P. San-Jose, Phys. Rev. Lett. 119, 107201 (2017).

[54] K. Seo, V. N. Kotov, and B. Uchoa, Phys. Rev. Lett. 122, 246402 (2019).

[55] T. Huang, L. Zhang, and T. Ma, Science Bulletin 64, 310 (2019).

[56] A. O. Sboychakov, A. V. Rozhkov, A. L. Rakhmanov, and F. Nori, Phys. Rev. B 102, 155142 (2020).

[57] J. Vahedi, R. Peters, A. Missaoui, A. Honecker, and G. Trambly de Laissardière, arXiv:2104.10694.

[58] A. Mielke, J. Phys. A: Math. Gen. 24, L73 (1991).

[59] A. Mielke and H. Tasaki, Commun. Math. Phys. 158, 341 (1993).

[60] A. Mielke, Phys. Lett. A 174, 443 (1993).

[61] R. Pons, A. Mielke, and T. Stauber, Phys. Rev. B 102, 235101 (2020).

[62] J. M. B. Lopes dos Santos, N. M. R. Peres, and A. H. Castro Neto, Phys. Rev. Lett. 99, 256802 (2007).

[63] E. J. Mele, Phys. Rev. B 81, 161405 (2010).

[64] A. L. Fetter and J. D. Walecka, Quantum Theory of ManyParticle Systems (McGraw-Hill, New York, 1971).

[65] R. E. Throckmorton and O. Vafek, Phys. Rev. B 86, 115447 (2012).

[66] J. M. Campanera, G. Savini, I. Suarez-Martinez, and M. I. Heggie, Phys. Rev. B 75, 235449 (2007).

[67] P. Moon and M. Koshino, Phys. Rev. B 87, 205404 (2013).

[68] J. M. B. Lopes dos Santos, N. M. R. Peres, and A. H. Castro Neto, Phys. Rev. B 86, 155449 (2012).

[69] R. Bistritzer and A. H. MacDonald, Proc. Natl. Acad. Sci. USA 108, 12233 (2011).

[70] T. Stauber, P. San-Jose, and L. Brey, New J. Phys. 15, 113050 (2013).

[71] T. Stauber and H. Kohler, Nano Lett. 16, 6844 (2016).

[72] J. Gonzalez, F. Guinea, and M. Vozmediano, Nucl. Phys. B 424, 595 (1994).

[73] R. Roldán and L. Brey, Phys. Rev. B 88, 115420 (2013).

[74] J. Sabio, J. Nilsson, and A. H. Castro Neto, Phys. Rev. B 78, 075410 (2008).

[75] T. Stauber, P. Parida, M. Trushin, M. V. Ulybyshev, D. L. Boyda, and J. Schliemann, Phys. Rev. Lett. 118, 266801 (2017). 